\title{
Hysteroscopic removal of angular retained products of conception hidden behind intrauterine adhesions using a 12-Fr spoon-shaped forceps
}

\author{
Aiqian Zhang, Huan Huang, Xian Qin, Arvind Burjoo, Nidhi, Dabao Xu, Chunxia Cheng \\ Department of Obstetrics and Gynecology, Third Xiangya Hospital of Central South University, Changsha 410013, China \\ Correspondence to: Dabao Xu, MD; Chunxia Cheng, MD. Department of Obstetrics and Gynecology, Third Xiangya Hospital of Central South \\ University, 138 Tongzipo Rd., Changsha 410013, China. Email: dabaoxu@yahoo.com; darlingch@126.com.
}

Submitted Dec 27, 2019. Accepted for publication Jan 14, 2020.

doi: $10.21037 /$ atm.2020.01.136

View this article at: http://dx.doi.org/10.21037/atm.2020.01.136

\section{Clinical vignette}

A 34-year old woman presented with failure to restore menstruation 4 months after the evacuation of retained products of conception (RPOC) following spontaneous abortion $(\mathrm{SAB})$ at 2 months gestation. Transvaginal 3-D ultrasound (Figure 1) showed that the endometrium of the middle and lower part of the uterine cavity was interrupted, and a $14 \times 11 \times 10 \mathrm{~mm}$ area of mixed echo mass could be seen in the left uterine cornu. The preoperative diagnosis was a left angular cavity retained products of conception accompanied by intrauterine adhesion (IUA).

\section{Surgical techniques}

\section{Preparation}

Lithotomy position was adopted and intravenous anesthesia was successfully administered after routine disinfection and draping in the operating room.

\section{Exposition}

Gynecological examination showed that the uterus was anteverted, and the cervix was exposed and disinfected. Sterile saline solution was used to distend the uterus at a distension pressure of $120 \mathrm{mmHg}$, with a flow rate of $350 \mathrm{~mL} / \mathrm{min}$. Hysteroscopy was performed under ultrasound monitoring.

\section{Operation}

Hysteroscopic adhesiolysis (HA) was performed using a blunt spreading dissection technique (1) with a 5-Fr double action spoon-shaped forceps, and then a 5-Fr single action cold scissors was used for further dissection of the adhesion and ploughing of the scar tissue (2). After the HA, the RPOC that in the left angular cavity and hidden behind the adhesion were successfully removed with ease using 12-Fr double-action spoon-shaped forceps (Figure $2 A$ ). A double-channel, 12-Foley catheter balloon (Figure 2B), with the top catheter portion beyond the balloon removed, was inserted into the uterine cavity and distended using $2.5 \mathrm{~mL}$ of sterile saline. Three $\mathrm{mL}$ hyaluronic acid gel was injected through the other channel of the Foley catheter into the uterine cavity.

\section{Completion}

The balloon was kept inside the uterine cavity for 2 weeks. Hormonal therapy started with estradiol valerate $3 \mathrm{mg}$ Bid for 28 days, and progesterone $200 \mathrm{mg}$ Qn was added for the last 6 days. A follow-up hysteroscopy was performed 1 month later to further manage the IUA. The procedure is shown in the Figure 3.

\section{Comments}

\section{Clinical results}

HA was successful, with the RPOC of the left angular cavity being completely removed in one setting, and the left angular cavity and tubal ostium being clearly exposed. There was no additional injury to the endometrium and 

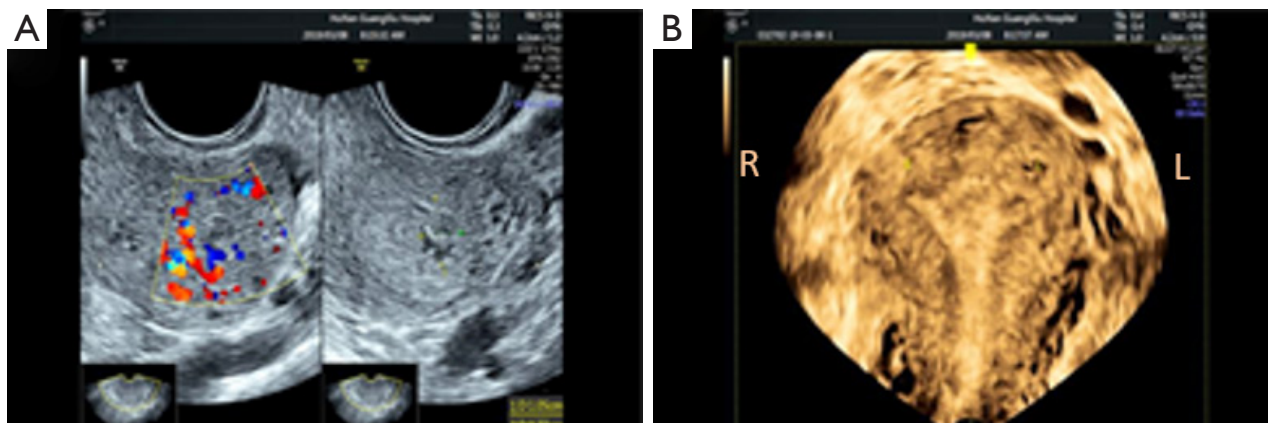

Figure 1 Transvaginal ultrasound image. (A) Two-dimensional ultrasound reveals uneven endometrium with loss of endometrial echo; (B) transvaginal 3-D ultrasound showed that the endometrium of the middle and lower part of the uterine cavity were interrupted, and a $14 \times 11 \times 10 \mathrm{~mm}$ area of mixed echo mass can be seen in the left uterine cornu.
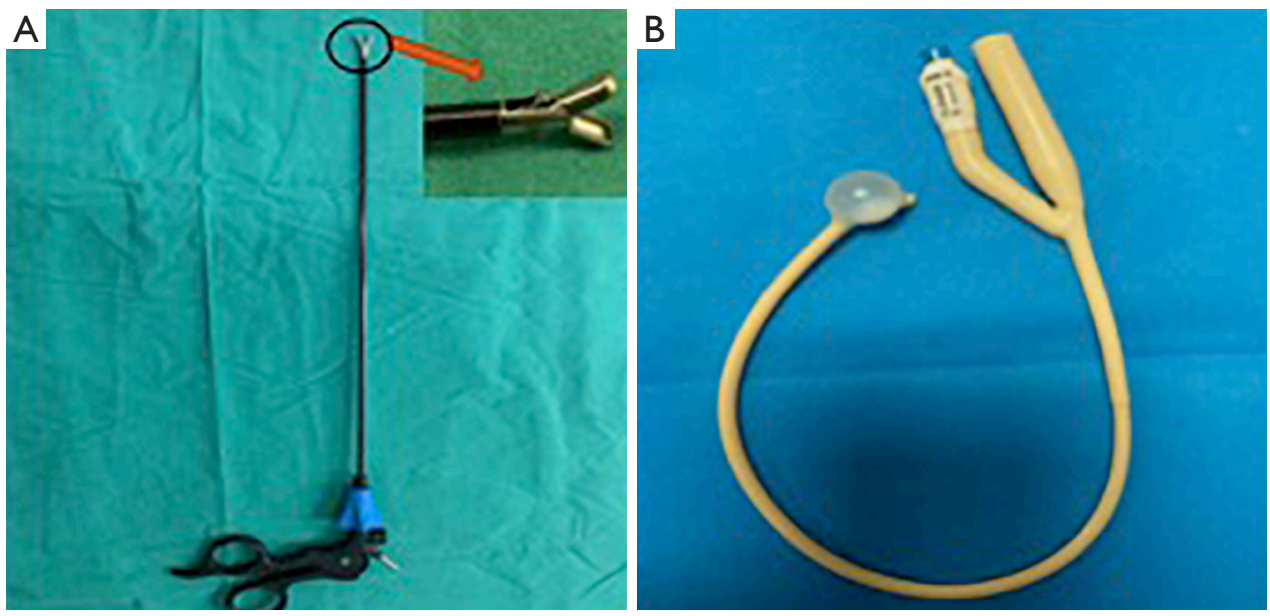

Figure 2 The typical instruments used. (A) A 12-Fr double-actions spoon-shaped forceps and an enlarged image of the spoon-shaped forceps; (B) a 12-Foley catheter balloon used for insertion into the uterine cavity.

no complications. The follow-up hysteroscopy 1 month later revealed an almost normal uterine cavity and with restoration of almost normal menses.

\section{Advantages}

It is usually difficult to completely remove RPOC in angular cavities on the first attempt, and this procedure entails a great risk of massive bleeding, uterine perforation, and fluid overload due to the particularly deep position, abundant blood supply, and weak muscular layer in the uterine horn. Moreover, multiple surgical operations may easily cause damage to the endometrium, which can lead to IUA that can seriously affect future fertility. There is no uniform standard for the surgical methods. Operation is more challenging when the RPOC are accompanied by severe IUA, especially when the remnants are hidden behind the adhesions. A hysteroscope with a large working channel, especially a 12Fr working channel with a double-action spoon-shaped forceps, has been used in clinical practice. The 12-Fr double action spoon-shaped forceps can grasp more RPOC tissue and is dramatically faster, stronger than traditional 5- or 7-Fr forceps. Moreover, it does not inflict electrical or thermal injury to the endometrium, and is thus more theoretically suitable for removing the RPOC in angular cavities. There were no reports regarding using a 12-Fr double-action spoonshaped forceps to remove RPOC of this type. The majority of the recently published papers describe removal of retained remnants using electrical instruments or curettage which may cause serious damage to the endometrium, especially in an angular cavity. This video of surgical procedure demonstrates the technique for hysteroscopic adhesiolysis using $5-\mathrm{Fr}$ 


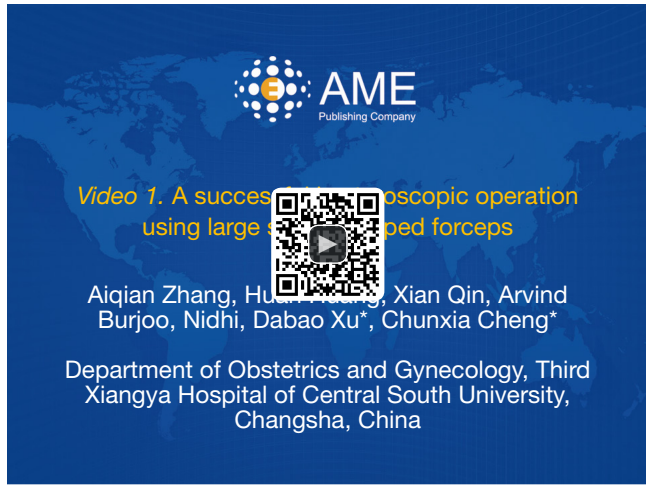

Figure 3 A successful hysteroscopic operation using large spoonshaped forceps (3).

Available online: http://www.asvide.com/watch/33099

forceps and scissors and highlights the advantages of using a 12-Fr double-action spoon-shaped forceps to remove RPOC in the left angular cavity accompanied by IUA.

\section{Caveats}

It is safe, fast, and effective to perform hysteroscopic adhesiolysis using 5-Fr forceps and cold scissors to first expose the RPOC in an angular cavity, and then to remove them using 12-Fr double-action spoon-shaped forceps. Moreover, the use of cold forceps provides good protection for the endometrium, especially for the angular parts. This is a new surgical technique and is worthy of further study.

\section{Acknowledgements}

Funding: This study was supported by Changsha Science

Cite this article as: Zhang A, Huang H, Qin X, Burjoo A, Nidhi, Xu D, Cheng C. Hysteroscopic removal of angular retained products of conception hidden behind intrauterine adhesions using a 12-Fr spoon-shaped forceps. Ann Transl Med 2020;8(4):59. doi: 10.21037/atm.2020.01.136 and Technology Bureau (Grant No. kq1901124).

\section{Footnote}

Conflicts of Interests: The authors have no conflicts of interest to declare.

Ethical Statement: The authors are accountable for all aspects of the work in ensuring that questions related to the accuracy or integrity of any part of the work are appropriately investigated and resolved. Approval was given to the study by The Institutional Review Board (IRB) of Third Xiangya Hospital of Central South University. The procedure was performed in accordance with relevant guidelines and regulations. Informed consent was obtained after the procedure was fully explained by all participants and their legal guardians.

\section{References}

1. Huang H, Cheng C, Johnson G, et al. Hysteroscopic Intrauterine Adhesiolysis Using a Blunt Spreading Dissection Technique With Double-action Forceps. J Minim Invasive Gynecol 2018;25:583-84.

2. Zhang A, Jamail G, Xue M, et al. Hysteroscopic Intrauterine Adhesiolysis Using the "Ploughing" Technique With Cold Scissors. J Minim Invasive Gynecol 2015;22:934-5.

3 Zhang A, Huang H, Qin X, et al. A successful hysteroscopic operation using large spoon-shaped forceps. Asvide 2020;7:059. Available online: http://www.asvide. com/watch/33099 